\title{
EL MERCADO DEL MAÍZ EN MÉXICO: DESDE EL ENFOQUE DE LA ECONOMÍA INDUSTRIAL Y LOS COSTOS DE TRANSACCIÓN
}

\author{
María de los Angeles Parra Vargas ${ }^{1}$, José G. Vargas-Hernández, M.B.A; Ph.D ${ }^{2 *}$, \\ 1 Maestría en Negocios y Estudios Económicos \\ Centro Universitario de Ciencias Económico Administrativas. Universidad de Guadalajara \\ 2 Profesor Investigador. Departamento de Administración \\ Centro Universitario de Ciencias Económico Administrativas. Universidad de Guadalajara. \\ Periférico Norte 799 Edificio G-201-7, Núcleo Universitario Los Belenes CUCEA \\ Zapopan, Jalisco C.P. 45100; México \\ Tel y fax: +52(33) 37703340, 37703300 ext. 25685 Email: jgvh0811@yahoo.com \\ jvargas2006@gmail.com
}

*Autor por correspondencia: josevargas@ cucea.udg.mx

Recibido: 24/05/2017

Aceptado: 31/07/2017

\section{Resumen}

El objetivo de la presente investigación es mostrar el papel que juegan las instituciones en mercados estratégicos para los países, en este caso el mercado del maíz en México. Comienza con una revisión de los antecedentes, esencialmente después de la entrada del TLCAN en México. Posteriormente el tema es abordado desde las grandes teorías que engloban a las instituciones para poder explicar factores que han hecho el mercado del maíz continúe operando en México. Explicando la manera actual de operar del mercado estudiando ante los costos de transacción, y por lo tanto las fallas del mercado. Concluyendo con la revisión del impulso que ha tenido la producción las instituciones rurales a través de algunos mecanismos como puede ser la producción por contrato en el medio rural.

Palabras clave: Mercado del maíz, economía institucional, costos de transacción, fallas del mercado.

\begin{abstract}
The objective of the present investigation is to show the role that the institutions play in strategic markets for the countries, in this case the maize market in Mexico. It begins with a review of the background, essentially after NAFTA's entry into Mexico. Subsequently the subject is approached from the great theories that cover the institutions to be able to explain factors that have made the corn market continue to operate in Mexico. Explaining the current way of operating the market by studying transaction costs, and therefore market failures. Concluding with the revision of the impulse that has had the production the rural institutions through some mechanisms such as the production by contract in the rural environment.
\end{abstract}


Keywords: Corn market, institutional economics, transaction costs, market failures. JEL: M21, R10, P30, L00, Q10

\section{Introducción}

Con el presente se pretende exponer los mecanismos comerciales presentes en el mercado del maíz en el Estado de Jalisco. Además de analizar el impacto que ha tenido la economía neoclásica y la economía institucional dentro de este mismo, analizar los fallos que están implícitos dentro del mercado; y conocer como las instituciones tienen impacto en este sector. La importancia del mercado del maíz puede comprobarse con las altas cantidades sembradas a nivel nacional de este grano básico. Entre los cultivos agropecuarios en México el cultivo de maíz blanco es el más importante a nivel nacional en términos de superficie, en la tabla de superficie sembrada por cultivo se observa que cuenta con $6,715,157$ de hectáreas sembradas seguido por el Sorgo grano con 2,199,230 hectáreas, diferencia significativa del uso de la tierra por cultivo. En el caso del maíz forrajero se observa una superficie sembrada con 225,302 ha, y en lo que corresponde a la Producción por cultivo.

Cultivo Anuales de México (Superficie sembrada, hectareas).

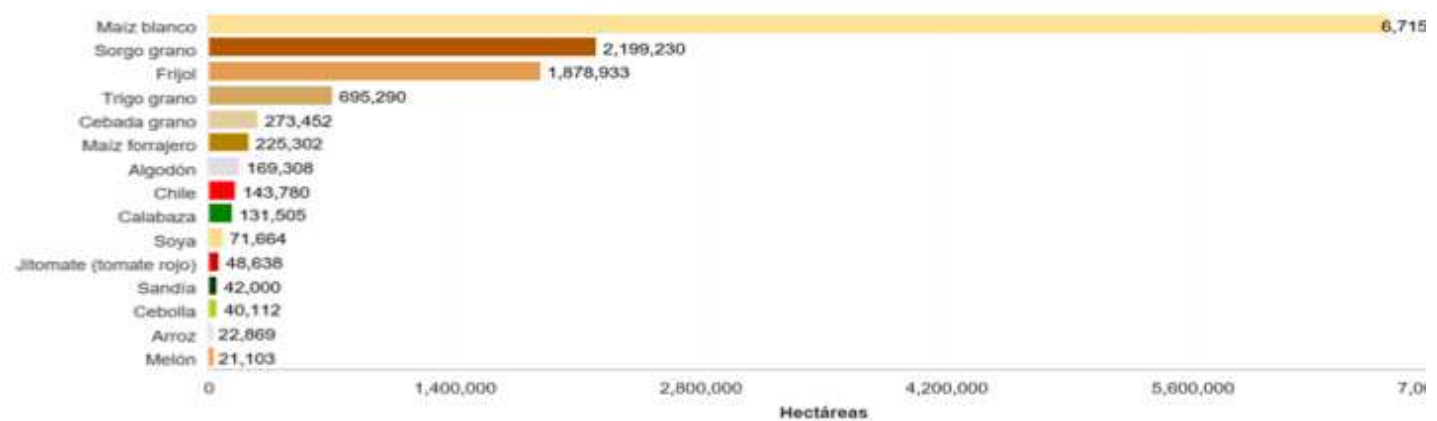

Figura 1

Fuente: INEGI (2014). Encuesta Naciona Agropecuaria 2014. Recuperado de:

http://www.inegi.org.mx/est/contenidos/proyectos/encuestas/agropecuarias/ena/ena2014. (Mayo 2017)

En el caso de los cultivos anuales por toneladas el maíz blanco ocupa el primer lugar de entre los cultivos a nivel nacional con 21,087,445 toneladas y el maíz forrajero con $5,675,285$. 

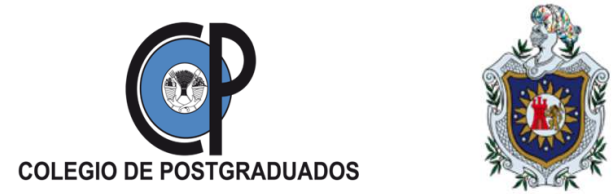

Revista Iberoamericana de Bioeconomia y Cambio Climático (Rev. iberoam. bioecon. cambio clim.)

Vol. 3 num 5, 2017, pag 752-768

ISSN eletrónico $2410-7980$

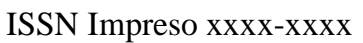

Cultivo Anuales de México (Superficie sembrada, toneladas).

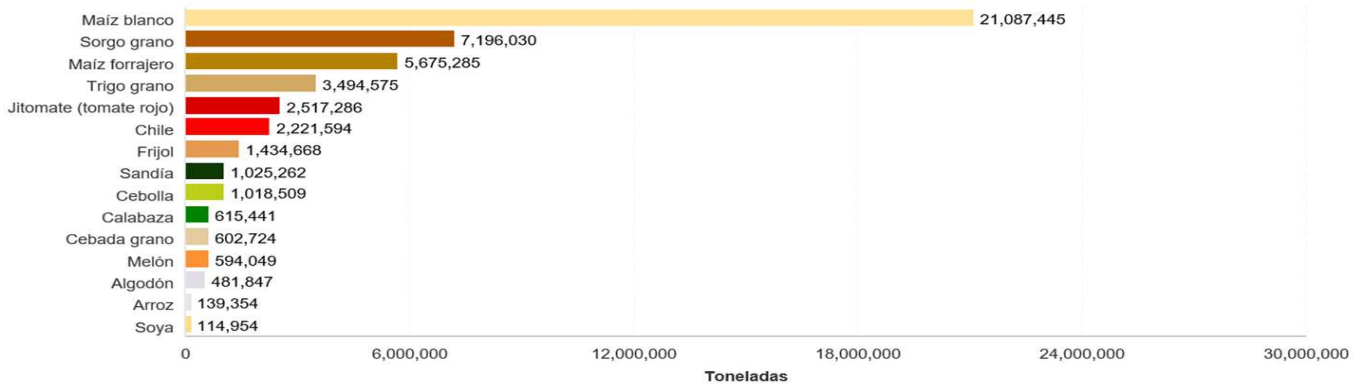

Figura 2

Fuente: INEGI (2014). Encuesta Naciona Agropecuaria 2014. Recuperado de:

http://www.inegi.org.mx/est/contenidos/proyectos/encuestas/agropecuarias/ena/ena2014. (Mayo 2017)

Aunque el Maíz es uno de los principales productos que más se producen en el país, se mantiene una gran dependencia al consumo internacional, pues ante cifras de SAGARPA JALISCO (2015), las importaciones en el 2015 fueron de 896 mil toneladas de Estados Unidos y en los años 2012 - 2015 el 56\% de las importaciones provino de Estados unidos y el otro $44 \%$ de Sudáfrica.

Por lo cual se puede observar que el mercado del maíz no es autosuficiente, sin embargo, para el presente año 2017, The New York Times ES reporto que cerca de 12,7 millones de toneladas son importadas, de maíz amarillo, que se usa principalmente para ganado y se complementa con las 3.5 millones de toneladas de maíz amarillo que se siembran en México. Exponen que México es autosuficiente en la producción de maíz blanco, produciendo 22,2 millones de toneladas en el año 2016, e importó cerca de 1,1 millones de toneladas de maíz blanco estadounidense para compensar las exportaciones rentables de este tipo de maíz a Sudáfrica y otros países (The New York Times ES., 2017).

Entre los usos que se le pueden dar al Maíz, que al mismo tiempo hacen muy importante a este mercado son los siguientes tres principales usos que nos marca la FAO (1993):

A. Como alimento, harina, gluten y aceites.

B. Como forraje.

C. Como materia prima para la industria como alcohol combustible, para la producción de bebidas, además el maíz se utiliza para mejorar los suelos (furfural y xilosa).

La FAO (1993), hace mención que: en el caso de los países localizados en aquellos llamados de desarrollo presentan producciones a escala, y se considera pronostica que el 2030 este poder de producción pasara de menos de la mitad que es actualmente a casi tres quintas partes. Lo anterior indica que la producción de maíz puede seguir en manos de países industrializados sin embargo es esencial determinar cuáles han sido las condiciones que mantienen adaptados a los pequeños agricultores en el mercado de maíz. 
El análisis puede ser visto desde el punto de vista del productor en el cual se desprotegido al mercado nacional, pero también puede ser retomado desde el perfil social en el cual las importaciones han traído un beneficio social en la reducción de precios, pero sin tomar en cuenta al Régimen de Protección Especial del Maíz las cuales son muy generales y con pocos criterios.

\section{Antecedentes del problema}

Existen estudios del mercado de maíz en México que analizan los cambios que ha sufrido el mercado del maíz desde la entrada del Tratado de Libre Comercio de América del Norte, en el caso de las regulaciones comerciales internacionales, García (2002) presenta entre la problemática, el cambio que existió de un mercado de sustituciones de importaciones al neoliberal en el cual el plan inicial para la protección de los productores mexicanos en lo que el país era capaz de integrarse al comercio internacional, en el que se encontraba considerado el sector agrícola, entonces en primera instancia fue la intención proteger a la economía mexicana en los primeros años de la entrada a este acuerdo comercial, sin embargo, las importaciones de maíz fueron en aumento debido a los bajos precios internacionales, sin poder impedir con eficacia este fenómeno aun con la devaluación del peso mexicano ante el dólar americano.

La planeación de las importaciones también juega un papel importante en la problemática del maíz, pues ha originado incremento en los costos de adquisición del grano debido a una mala planeación de estas mimas, y el no contar con este plan también afecta al almacenaje del grano pues no se toma en cuenta la temporada de cosecha en el país y aumenta significativamente el costo del almacenaje.

El objetivo particular al que pretende llegar García (2002) con su investigación es el de analizar la eficacia de la tasa arancelaria del impuesto general de importación con el objeto de determinar la protección del mercado del maíz ante la entrada del TLC, entonces plantea que la tasa arancelaria del impuesto general de importación es un instrumento de protección del mercado del maíz. Y su aportación a este campo es que un arancel del $3 \%$ no es suficiente para la protección del mercado y que uno de $45 \%$ habría ayudado a poner a las importaciones en un nivel semejante a la cuota libre de arancel establecida en el TLCAN (García, 2002: 1$5)$.

Por otro lado, continuando con la línea de investigación de García, el análisis visto con la no protección a los productores de maíz se planteó en el beneficio al consumidor, en cual se reduce el excedente del productor, pues además de los precios competitivos a los que se tuvieron que enfrentarse los productores de maíz desaparecieron los programas de apoyo que se brindaban por parte de PROCAMPO ahora PROAGRO, y por lo tanto menores subsidios para la producción mexicana. Entre la problemática planteada por García y Williams (2004) presenta una mala política comercial. Sin embargo y a pesar de la alta 


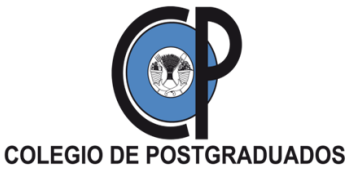

Revista Iberoamericana de Bioeconomia y Cambio Climático (Rev. iberoam. bioecon. cambio clim.) Vol. 3 num 5, 2017, pag 752-768 ISSN eletrónico 2410-7980

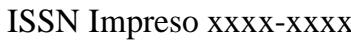

producción de maíz alcanzada en el periodo de vigencia del TLCAN, las importaciones del grano fueron mayores a la cuota establecida en el Tratado.

Entre los fenómenos pudieron observarse una elevación en el consumo, bajos precios internacionales de 1994 y bajo tipo de cambio, también menciona el autor fueron algunos los anteriores fenómenos fueron unos factores que explican el incremento de las compras al exterior. Entre los resultados de este nuevo estudio se pudo identificar que los perdedores de la política de mayor apertura comercial fueron los productores. Pues no se respetó la política comercial establecida en el TLCAN y por consecuencia la producción de maíz fue menor. Menciona la posibilidad de que las superficies que no fueron sembradas con maíz se hayan destinado a otros cultivos que les fueras más redituables a los productores y dueños de la tierra, como ejemplo hortalizas.

Los precios más bajos en el modelo neoliberal generaron un consumo mayor, y por lo tanto la disminución de precios tuvo efectos anti-inflacionarios. También señala que una de las importantes características del mercado analizado es dado por su utilización como ingrediente en la alimentación humana y animal de México, y su presencia en más de dos terceras partes de los productores agrícolas, por lo tanto, también llega a la conclusión de que el maíz es el cultivo más importante en el sector agrícola de México.

Por su importancia, el maíz y como nos menciona García y Williams (2004) este grano tiene efectos multiplicadores en el resto de la economía, es decir que un aumento en su precio puede tener efectos en la inflación del sector agrícola y en la economía del país, por lo cual es importante no dejar de lado este análisis y prestar a las señales que el sistema de precios otorga a este mercado.

No se debe olvidar la estrategia seguida por el Estado en la que en el tercer año de entrada en vigor del TLCAN los elaboradores de la política agrícola del gobierno decidieron eliminar la protección del maíz que se había negociado mantener hasta el año 2008. Por lo cual la soberanía y autosuficiencia alimentaria dejaba de ser un meta, para poder estar dentro del marco del libre comercio, en donde el Estado deja todo a la globalización, confiando en sus posibles implicaciones, como puede ser el caso de los altos costos de transporte de los puertos de salida de los Estados Unidos a los centros de consumo de México, que nos señala García y Williams (2004) y a la baja elasticidad precio de la oferta y demanda de maíz, se espera que los efectos de la liberación total en la producción, el consumo y las importaciones no sean significativos (García y Williams, 2004: 169-213).

Desde el punto de vista de las consecuencias de la globalización nos plantea Eakin, Perales, Appendini, \& Sweeney (2014) la problemática que han afectado significativamente a los pequeños productores quienes no cuentan con la tecnología necesaria para el volumen de producción que les genere una ventaja competitiva para poder competir con los productores de los países internacionales. Lo anterior tomando en cuenta sus factores internos de producción. El impacto del neoliberalismo y del TLCAN en los pequeños de la producción de maíz ha sido ampliamente concebida como negativa, asociados con la degradación 
ecológica, la emigración rural emigración y la erosión cultural. Presentando al mismo tiempo la existencia de pequeños productores aislados a pesar de las fuertes economías agrícolas que tienen sistemas de gran escala en la producción que atacan los mercados comerciales.

Sin embargo, los pocos productores de maíz que han permanecido en el campo son mayormente pequeños propietarios, mismos que han permanecido constantes en el mercado aun a pesar de las políticas desfavorables por las que se han visto afectados, y de la evolución de las estructuras agrícolas de gran escala. Por lo cual es observable la capacidad de adaptación en el mercado de la agricultura del maíz mexicano. La manera de su comercialización se ha visto reflejada en pequeños vendedores que venden sus productos a intermediarios o en su caso a minoristas y para su consumo familiar. Lo cual ha ayudado en la participación de estos pequeños propietarios en procesos de desarrollo económico, políticas públicas y la asignación de los recursos para la maximización de la eficiencia pública y bienestar social. Importancia de la planificación del desarrollo y el contexto sobre el que se desenvuelve el maíz es la seguridad alimentaria, la cultura y la política (Eakin et al. 2014: 133 - 135).

En cuanto a los subsidios otorgados por las instituciones mexicanas y el funcionamiento de la política agrícola del gobierno mexicano exponen Fox, \& Haight (2010) los autores exponen que se encuentran desequilibradas las características de los productores de maíz, dejando de beneficiar a los productores de bajos ingresos, dando prioridad a los grandes agricultores. El acceso para la nueva generación de programas de apoyo tiene normalmente elevados costos de transacción: los agricultores necesitan organizarse entre ellos y presentar la documentación necesaria para aplicar al programa. Para muchos de los pequeños productores agrícolas, estos gastos han sido excluyentes. Los grandes productores con buena infraestructura y una orientación comercial han tenido más ventajas para ser beneficiarios de estos programas durante el último decenio (Fox et al. 2010: 11-12).

Ante el análisis de la entrada en vigor del tratado de libre comercio y por lo tanto la desprotección del campo por la entrada al sistema neoliberal, podemos entonces pasar a analizar los factores que han sido importantes para que sigan vigentes los productores del campo siendo en total de acuerdo a cifras del censo de agricultura de México, existen más de 2,8 millones de agricultores de maíz en el país (INEGI, 2014).

\section{Justificación}

Las contribuciones que este análisis brindará y servirá para conocer el contexto actual del mercado del maíz en México, pues es un importante sector del cual no solo depende un sinfín de empleos, sino que también depende su seguridad alimentaria, por lo que es conveniente analizar la problemática a detalle. También será de impacto debido a la poca información actual sobre el tema, en donde hay pocas investigaciones recientes. Tanto en el ámbito de las políticas públicas, comerciales como retomado desde el contexto de la economía institucional. 
El método de investigación utilizado en el presente es uno de correlación para determinar la relación entre las variables. Y cualitativo para la exploración de la documentación y análisis.

\section{Marco teórico}

Coase (1988) expone que, si se quiere pasar de un régimen de cero costos de transacción a uno de costos de transacción positivos, lo que se refleja a primera instancia es la importancia crucial del sistema jurídico. Marcando la importancia de especificar el marco institucional en el cual se llevará a cabo la negociación para el intercambio. Puesto que el entorno institucional afecta a los incentivos económicos para producir en la economía los costos que implican las transacciones, en este caso los intercambios o compra de venta de productos.

De acuerdo a Mahoney and Rajendran Pandian (1992) entonces la teoría de los costos de transacción ayuda a explicar y predecir la gobernanza para desarrollar la eficiencia de la organización. También hace énfasis en los compromisos creíbles los cuales expone son vistos como formas de protección contra riesgos contractuales debido a que es natural contar con contratos incompletos.

En el caso de los beneficios económicos que pueden obtenerse de la cooperación Mahoney (2012) considera dos puntos importantes los cuales son: 1.- las personas son diferentes y por lo tanto tendrán talentos diferentes, y 2.- la especialización en las tareas mejorará la eficiencia con que llevan a cabo sus actividades los individuos. Entonces es importante la cooperación para poder obtener una asociación entre las partes interesadas, en este caso en los mercados.

En el caso de Arrow (1974) toca la vertiente de las propuestas para los incrementos de los salarios mínimos. Ante la irregularidad en la distribución de la riqueza puede pensarse que es cuestión de solo un aumento en los salarios. Sin embargo, el sistema puede reaccionar a esa política. Los resultados pueden ser una tendencia sustituir el trabajo por capital y las consecuencias elevados volúmenes de desempleo, consecuencia económica que puede ser peor que salarios bajos.

Económicamente es bien vista la función importante que hace el sistema de precios quien realiza una eficiente labor en la asignación de los recursos. Arrow (1974) menciona que con dicho sistema los actores tienen libertad para actuar en el sistema, sin embargo, los ingresos no se encuentran diversificados por la justicia, sino más bien este sistema sufre de un sentido ético.

De acuerdo a Mahoney (2012) es importante notar la eficiencia económica que se logra a través de este sistema. Sin embargo, este sistema de precios no siempre funciona, 

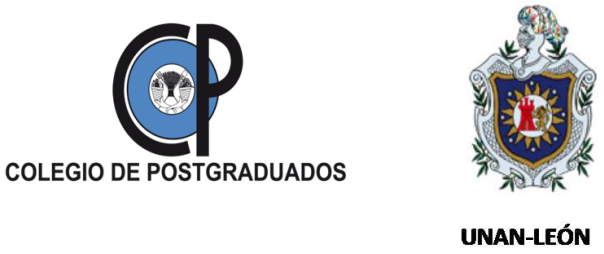

Revista Iberoamericana de Bioeconomia y Cambio Climático

(Rev. iberoam. bioecon. cambio clim.)

Vol. 3 num 5, 2017, pag 752-768

ISSN eletrónico 2410-7980

ISSN Impreso xxxx-xxxx

pues existirán implicaciones que ocasionaran en este sistema fallos en los que el mismo sistema no podrá participar para su solución.

Entonces ¿Qué es lo que podría evitar estas fallas del mercado, de manera óptima? Arrow (1974) responde a esta pregunta afirmando que la confianza ayudará al sistema social en gran medida, aunque esta al igual que valores similares no sea fácil de conseguir. De ser fácil de adquirirla entonces habría menores problemas en el mercado, y por lo tanto menos implicaciones que generadas por la asimetría de la información, que crean ineficiencia en el mercado. En donde la falta de conciencia social es pérdida económica.

Mahoney (2012) expone un punto muy importante dentro de la economía en el que externalidades positivas como la Confianza y valores similares, lealtad, tienen un alto valor económico; con el que se eleva la eficiencia del sistema, permiten a los individuos producir más productos entre otros beneficios. Sin embargo, estas externalidades positivas no son básicos, pero sí de gran ayuda. Entonces desde la perspectiva de la justicia distributiva, y un análisis de gobernanza quien es el gobierno en todos sus niveles. El Gobierno participa en la asignación de recursos por medios que operan dentro del sistema de precios, para corregir los fallos que pudieran surgir. Entonces el gobierno tiene su serie de leyes y reglamentos, que pueden ayudar a controlar el mercado, la economía y la sociedad en general (Mahoney, 2012)

Entonces es aquí donde es bien vista la intervención del Estado, en solucionar externalidades negativas que puedan afectar al sistema o a la sociedad. Es decir, solucionar las fallas que simplemente el sistema de precios no pudo solucionar (Varian, 2010). Es de suma importancia destacar para este estudio lo que sostiene Arrow (1974) donde el canal para conseguir beneficios son las organizaciones mediante acciones colectivas, esto ante aquellas circunstancias en las que el sistema de precios emita una falla al sistema.

Arrow (1974) menciona una falla más del sistema de precios en la que hace referencia a la presencia de incertidumbre. Entonces si la incertidumbre se conoce, podría afectar a los deseos de los individuos para el comercio. Lo que da como consecuencia que en lugar de contratos para comprar y vender cantidades fijas de mercancías, sería mejor tener contratos condicionales, o contratos en contingente de productos básicos. Sin embargo, si por el hecho de contar con los contratos para comprar y vender cantidades fijas se entra en el área de los costos de transacción, el hacer contratos condicionales elevaría aún más los costos, haciendo imposible realizarlos pues debido a la incertidumbre existiría una lista compleja de precios para este tipo de contratos, y al mismo tiempo estará limitada la capacidad de los individuos para comprender este tipo de contratos. "Entonces en las contingencias para las cuales hay disponibles contratos condicionales es mucho más limitado de lo que sería deseable en teoría idealmente.

Coase (1960) propone que, si los costos de transacción son cero, entonces la ley no tendría el propósito de servir a la eficiencia económica. También sostiene que en el caso de la empresa y el mercado estos serán la estructura institucional del sistema económico. De esta manera aborda la definición de los costes de transacción a quienes define como aquellos 


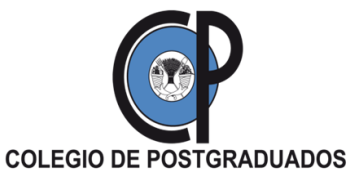

Revista Iberoamericana de Bioeconomia y Cambio Climático (Rev. iberoam. bioecon. cambio clim.) Vol. 3 num 5, 2017, pag 752-768 ISSN eletrónico 2410-7980 ISSN Impreso xxxx-xxxx

que ayudan a explicar por qué la empresa existe. Entonces los costos de transacción incluirán: 1.- Costos derivados de información y búsqueda; 2.- Negociación y decisiones; y c) Gastos por vigilancia y monitoreo. Entonces Coase (1988) presenta que las empresas que surgen para organizar aquellas transacciones de mercado que cuentan con fallos, podrán emerger siempre que sus costos sean inferiores a los costos de realizar transacciones a través del mercado.

Mahoney (2012) define a los mercados como instituciones cuyo objetivo es facilitar el intercambio, es decir, su función principal es reducir los costos de llevar a cabo las transacciones donde se encuentra presente el intercambio. Entonces la teoría económica, expone que, si los costos de transacción son inexistentes, los mercados no tienen ninguna razón de ser. En el caso de las limitaciones del sistema de precios para la asignación de los recursos, el riesgo moral es definido como los precios de riesgo en la dificultad de distinguir entre aquellos riesgos y fracasos que son para optimizar. En donde las personas pueden cambiar de comportamiento, por ejemplo, al contratar una póliza de seguro, o entrar en una relación laboral.

Arrow (1971) expone el caso anterior también es conocido como seguros selección adversa, entonces el asegurado conocerá más sobre sus riesgos que la aseguradora, la aseguradora podrá establecer los precios para estos seguros, sin embargo los individuos con mayor riesgo serán más propensos a contratar los seguros que aquellos que no lo son, entonces para fines de permanencia de las aseguradoras tendrán que tener tasas elevadas, sin embargo esta tasa alta será para los dos grupos de solicitantes del servicio. Entonces de esta manera en el mercado de los seguros habrá muchos con riesgos que no están cubiertos de manera óptima. Entonces en estos casos las imperfecciones o fallas con que cuenta el mercado es debido a la asimetría de la información entre las dos partes que celebran el contrato. Akerlof (1970) sostiene que la asimetría de información limitara la existencia de contratos que den protección contra el fracaso donde el agente pueda desarrollar negocios exitosos.

\section{Revisión de la literatura empírica}

Como se ha expuesto, ante las desproporciones de las características con que cuentan los productores de maíz podemos observar que muchos de ellos por el hecho de contar con pocas hectáreas, bajos ingresos, tienen que recurrir a organizaciones de productores con las cuales puedan cumplir sus objetivos ante la desprotección que enfrentan por parte de poco control de las instituciones en las económicas emergentes como es el caso de México (Vargas y Guerra, 2014).

Lo anterior ligado como es el caso de los apoyos al campo extranjero como nos expone García (2002: 13-14) que "es un factor que resta competitividad a la producción del maíz en México". Es decir; entre los apoyos a otros países que brindan a sus productores 
agrícolas a través de los subsidios, destaca el caso de Japón, la Unión Europea y los Estados Unidos quiénes dan considerables cantidades de subsidios a productos agrícolas por el hecho de ser considerados como estratégicos para sus economías. En el caso de Japón, este ha aumentado el monto de subsidios para la producción de arroz, grano que se encuentra en su dieta principal, en los casos de la UE son la leche y trigo.

Camiro, Altamirano y Rojas (2009) presentan un estudio de caso de la intermediación financiera en el sur de Sonora, México. En el cual relaciona la problemática del mercado del maíz con poco marco jurídico, políticas no favorables, acceso limitado a instituciones financieras para obtener créditos de mediano y largo plazo, segmentación del mercado, pocos intermediarios, poca oferta de servicios y falta de incentivos para mejorar su calidad, así como altos costos de transacción.

La falta de intermediarios relacionada con las carteras vencidas con las que muchas de estas empresas cuentan o que terminan haciéndolos salir del mercado es decir termina afectando a la parafinanciera para que ya no cuente con los recursos necesarios para poder acceder al crédito otorgado por las instituciones competentes, en este caso FIRA.

Entre los datos expuestos del estudio anterior se mencionan los costos de producción que hubo sectores que de 1991 a 1996 mismo que impactaron de manera negativa con mayor fuerza; como la tasa de interés, los fertilizantes, disminución en la renta de la tierra y en el pago de salarios. En contraparte, los precios entre 1996 y 1998, cuando los cultivos principales se redujeron.

\section{Marco contextual}

Por los antecedentes expuestos es interesante analizar los posibles factores que influyen en la permanencia de los productores minoristas a pesar de las condiciones desfavorables expuesta. Entre las características que se pueden observar es necesarios exponer a los apoyos por volumen sembrado (PROAGRO antes PROCAMPO). Los intermediarios financieros rurales y otros organismos que han contribuido a la permanencia de productores agrícolas.

En este apartado se analizan las contribuciones que realiza al sector la empresa Productores Agropecuarios de Tlajomulco SPR de RL, ubicada en la colonia Lomas de Tejeda, en Tlajomulco de Zúñiga, Jalisco; siendo esta misma una organización que inicia operaciones en 1995, creada con el objetivo de apoyar a la solución de problemas relacionados con la comercialización, acceso al financiamiento y baja rentabilidad en la producción de maíz. En relación a otros servicios que ofrece la organización como el de apoyo a la comercialización de maíz, se han gestionado operaciones de agricultura por contrato para 20,000 toneladas en promedio, lo que garantiza acceso a mejores condiciones de venta del grano; las compras en volumen de insumos agrícolas es otro de los beneficios hacia sus socios.

Productores Agropecuarios de Tlajomulco, SPR de RL (2017) 


\section{MISIÓN}

Proporcionar soluciones integrales a nuestros socios a través de servicios de financiamiento, asistencia técnica y dispersión de riesgos, con el objetivo de mejorar la rentabilidad de sus unidades productivas.

\section{VISIÓN 2020}

Consolidarse en el mercado de oferta de servicios de financiamiento de granos básicos para una superficie de 250,000 hectáreas en la zona centro del estado de Jalisco, así como diversificar la oferta de productos de crédito.

Apoyan significativamente a la agricultura por contrato y el aseguramiento de las superficies sembradas; Según 20 Minutos (2017) el esquema de Agricultura por Contrato. Es la operación por la que el productor vende al comprador antes de cosechar su producto y se enmarca en uno de los mecanismos institucionales más efectivos para lograr la integración de los productores con redes de valor y poder acceder a los beneficios de los mercados.

En este caso FIRA quien realiza funciones como facilitador del acceso al crédito por medio de operaciones de crédito y descuento, garantías de crédito a proyectos relacionados con la agricultura, ganadería, avicultura, agroindustria, pesca o afines que se realizan en el medio rural (FIRA, 2016).

En Jalisco FIRA apoya de manera muy significativa al maíz; poco más de $25 \%$ del total de crédito FIRA es para el apoyo a la productividad del maíz en todas sus etapas (EL ECONOMISTA, 2014).

\section{Tabla 1}

Programas ofrecidos por FIRA al cultivo de maiz mexicano.

Programas otorgados por FIRA a la Red de Producción del Maiz

\begin{tabular}{ll} 
Programas & Objetivo \\
Profertil & $\begin{array}{l}\text { Crédito para el abasto de insumos estratégicos como semillas y fertilizantes en } \\
\text { compras organizadas y con anticipación al ciclo de siembras. }\end{array}$ \\
Avios Agricolas & $\begin{array}{l}\text { Para cubrir capital de trabajo del ciclo de producción, conforme a un paquete } \\
\text { tecnológico definido. }\end{array}$ \\
Créditos Refaccionarios & Para mejoramiento de suelos, maquinaria, equipo especializado de siembra, \\
\hline
\end{tabular}

Elaboración propia en base a: El Economista (2014). Recuperado de

http:/eleconomista.com.mx/columnas/agro-negocios/2014/04/01/importancia-retos-actuales-maiz-jalisco-

El monto de los prestamos está constituido como sigue: 
Tabla 2

Monto de Crédito por Acreditado Final Nacional

\begin{tabular}{ll}
\hline Estrato & Monto por Acreditado Final UDIS \\
\hline Microempresa & \\
Empresa Familiar & Hasta 33,000 \\
Pequeña Empresa & Hasta 160,000 \\
Mediana Empresa & Hasta 4,000,000 \\
Empresa Grande & Más de 4,000,000 \\
\hline
\end{tabular}

Fuente: Elaboración propia con base a FIRA. (2016). Credito FIRA. Recuperado de: http://www.fira.gob.

Quien brinda esos financiamientos a los intermediarios rurales para que estos a su vez puedan financiar a los agricultores mexicanos con el fin de que inviertan esos créditos en compra de insumos para la siembra de temporada, maquinaria, entre otros. Los beneficios por parte de FIRA muchas de las veces son utilizados para comprar los insumos requeridos para la siembra, del ciclo en el que siembran las fechas, pagando a las financieras a la hora de cosechar para vender a la comercializadora. También los intermediarios pueden brindar crédito para la compra de maquinaria. Los anteriores siendo unos de los usos más comunes para los agricultores.

Otro de los programas que apoyan al mercado del maíz es SAGARPA y uno de los programas que ofrecen es PROAGRO antes PROCAMPO. El objetivo de PROAGRO es dar liquidez a las Unidades Económicas Rurales y Agrícolas para invertir en actividades productivas SAGARPA (2016).

El apoyo que ellos reciben de acuerdo a PROAGRO está determinado según los siguientes montos máximos de incentivo por solicitante (UERA conformada por la superficie de los predios agrícolas objeto de dicho incentivo que posee un productor) por ciclo agrícola, será el correspondiente a 80 ha por productor ya sea como persona física o como integrante de una persona moral, conforme a las cuotas por ha siguientes:

Tabla 3

Cuotas pagadas por PROAGRO a productores de maiz mexicano

\begin{tabular}{lrr}
\hline Estrato & & Cuota por hectárea \\
\hline & Productores con UERA de hasta 3 hectáreas de temporal & 1500 \\
Auto-Consumo & Resto del estrato de autoconsumo & 1300 \\
Transición & & 750 \\
& & 450 \\
& & 180 \\
\hline & Productores con UERA de 20 ha hasta 50 ha de temporal y aquellas de más de 5 1 \\
Comercial & Resto del estrato comercial & \\
Fuente: Elaboración propia con base a SAGARPA. (2016). Reglas de operación. Recuperado de: & \\
www.sagarpa.gob.mx/agricultura/Programas/proagro/Normatividad/Documents/2017/2016_12_31_dof_rop_fomento_a_- &
\end{tabular}


Revista Iberoamericana de Bioeconomia y Cambio Climático (Rev. iberoam. bioecon. cambio clim.)

Vol. 3 num 5, 2017, pag 752-768

ISSN eletrónico 2410-7980

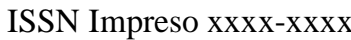

Como se puede ver el apoyo por parte de PROAGRO, hace una distribución de acuerdo al ciclo de siembra y a la cantidad de hectáreas con que cuenta cada productor, aun cuando la producción sea solo para autoconsumo, siendo aquí el apoyo limitado por la naturaleza de la clasificación. En el caso del estrato comercial, es apoyo disminuye por hectárea, pero considerando que regularmente en esta clasificación entran grandes productores el apoyo puede ser pronunciando, también es importante considerar que existen restricciones en cuanto a superficie.

En el caso de la producción por contrato, de acuerdo al entorno que rodea al intermediario financiero rural caso, se presenta tabla en la que se muestran los municipios con mayor volumen de producción y el volumen de unidades que reporta producción bajo contrato:

\begin{tabular}{|c|c|c|c|}
\hline \multicolumn{4}{|c|}{ Productividad del maiz y agricultura por contrato en México (Maiz blanco) } \\
\hline La Barca & $194,144.54$ & 2,602 & 57.00 \\
\hline San Juan de los Lagos & $109,151.93$ & 763 & No disponible \\
\hline Ameca & $108,540.33$ & 2,624 & 664.00 \\
\hline Tototlán & $100,423.91$ & 967 & 8.00 \\
\hline Tlajomulco de Zúñiga & $90,463.58$ & 1,736 & 27.00 \\
\hline Ocotlán & $84,581.59$ & 965 & 23.00 \\
\hline Atotonilco el Alto & $80,483.13$ & 1,217 & 37.00 \\
\hline San Martin Hidalgo & $76,007.68$ & 1,449 & 175.00 \\
\hline Ayotlán & $63,402.63$ & 1,204 & 24.00 \\
\hline Tepatitlán de Morelos & $58,010.21$ & 734 & 11.00 \\
\hline
\end{tabular}

En el caso del maíz blanco y del municipio que más produce maíz de acuerdo al último censo agrícola la Barca se observa que es el principal productor y sin embargo no

Tabla 5

Productividad del maiz y agricultura por contrato en México (Maiz amarillo)

\begin{tabular}{|c|c|c|c|}
\hline Maíz amarillo & Producción obtenida (Toneladas) & Unidades de producción & Unidades de producción que reportan agricultura bajo contrato \\
\hline Tepatitlán de Morelos & 67273.35 & 771 & 11 \\
\hline Arandas & $51,897.88$ & 928 & 57 \\
\hline San Miguel el Alto & $37,784.89$ & 445 & No disponible \\
\hline Encarnación de Diaz & $32,206.62$ & 172 & No disponible \\
\hline San Ignacio Cerro Gordo & $23,594.88$ & 446 & No disponible \\
\hline Atotonilco el Alto & $22,200.85$ & 271 & 37.00 \\
\hline Jesús Maria & $17,630.81$ & 485 & 18 \\
\hline Zapotlanejo & $13,628.18$ & 250 & 5.00 \\
\hline Quitupan & $12,727.26$ & 499 & No disponible \\
\hline Tototlán & $9,674.70$ & 97 & 8.00 \\
\hline Totatiche & $9,671.77$ & 374 & No disponible \\
\hline Atengo & $9,607.26$ & 202 & 3.00 \\
\hline Concepción de Buenos Aires & $9,169.09$ & 302 & 3.00 \\
\hline Tomatlán & $9,098.84$ & 564 & 20.00 \\
\hline Mascota & $8,936.42$ & 434 & No disponible \\
\hline Total Jalisco & 647322.67 & 19612 & 7368 \\
\hline
\end{tabular}

Fuente: Elaboración propia con base a: INEGI, Censo Agricola, Ganadero y Forestal 2007. (Mayo, 2017) 

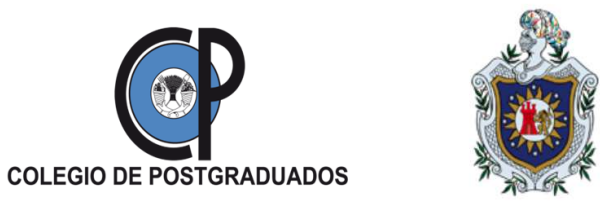

UNAN-LEÓN
Revista Iberoamericana de Bioeconomia y Cambio Climático (Rev. iberoam. bioecon. cambio clim.) Vol. 3 num 5, 2017, pag 752-768 ISSN eletrónico 2410-7980

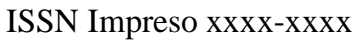

reporta el mayor número de unidades en agricultura bajo contrato en el caso del maíz blanco. Quien más reporta unidades de producción bajo el esquema de contrato es Ameca, ocupando el tercer lugar de producción obtenida.

En el caso del maíz amarillo, se reportan de acuerdo a cifras del INEGI (2007) 11 unidades de agricultura bajo contrato por Tepatitlán quien más siembra de este tipo de maíz, sin embargo, el segundo lugar Arandas es quien cuenta con el mayor número de unidades bajo contrato.

Se cuenta con limitaciones en la información pues existe información no disponible, además de que ya ha pasado un largo tiempo desde el último censo agrícola. La información anterior servirá para tomar en cuenta que las instituciones y la manera de operar del mercado de origen serán determinantes clave en la manera de comercializar.

\section{Conclusiones}

Por el marco teórico es posible analizar la existencia de las organizaciones de productores ya que el sistema neoliberal no es capaz de dar un equilibrio en el mercado del maíz, pues las cantidades de la producción en el campo está condicionada por instituciones encargadas de regular la economía nacional. Entonces aun a pasar de dejar la soberanía alimentaria de lado como expone García y Williams (2004) podemos observar que ha sido importante el papel de las organizaciones que están designadas para el apoyo del mercado del maíz en este caso los intermediarios financieros rurales.

Después de la desaparición de BANRURAL, en donde esta institución tuvo problemas por la ineficiencia, de acuerdo a Vargas (2014) las empresas propiedad de gobierno tienden a ser ineficientes, pues no tendrán incentivos para operar eficaz. Entonces con el fracaso de BANRURAL y Financiera Rural surge Financiera Nacional de Desarrollo Agropecuario, Rural, Forestal y Pesquero, quien en conjunto con Fideicomisos Instituidos en Relación con la Agricultura (FIRA), Agencia de Servicios a la Comercialización y Desarrollo de Mercados Agropecuarios (ASERCA), Secretaría de Agricultura, Ganadería, Desarrollo Rural, Pesca y Alimentación (SAGARPA) se han encargado de mantener regulado el mercado del maíz.

Es aquí donde la participación del Estado es bien vista de acuerdo a lo mencionado por (Varian, 2010) para solucionar fallos del mercado, pues existe infinita asimetría de la información debido a oportunismo en el medio, pero principalmente a los orígenes de las instituciones en las economías emergentes debido a la falta de confianza, asimetrías de información e instituciones débiles, por lo tanto, existe el ejercicio del oportunismo Vargas (2014). Entonces por lo anterior y en conjunto con las políticas de libre comercio presentes a inicios de los años noventa, surgen las instituciones financieras rurales designadas y apoyados por FIRA para formar organizaciones de los productores, en los cuales no cuentan solo con servicios financieros si no también apoyan como intermediarios entre los productores de maíz y los compradores finales como pueden ser MASECA, MINSA, por mencionar a algunos. 
Entonces es donde de acuerdo a Mahoney (2012) los individuos pueden decidir poner su confianza o buscar en constantes ocasiones este valor que promete eficiencia en el sistema. Por lo cual Arrow (1974) propone que el objetivo de las organizaciones es ayudar a conseguir beneficios en donde el colectivismo en circunstancias donde el sistema de precios da una falla de mercado. De acuerdo a las aportaciones de Coase (1988) quien presenta que las empresas que surgen para organizar aquellas transacciones de mercado que cuentan con fallos, mientras que sus costes sean inferiores a los de transacción del mercado.

Mientras que a nivel internacional existen instituciones constituidas por el mismo sistema actual neoliberal en el que el mismo sistema de precios es el encargado de determinar los precios a nivel mundial como es el caso de la bolsa de chicago quien funge como regular de precios a nivel mundial.

FIRA (2015) presenta que el maíz en la actualidad está atravesando por un periodo de gran recuperación, pues ha habido un aumento en los inventarios internacionales y por lo tanto la caída de los precios, a partir del 2015, existiría reducción en la siembra del maíz debido a los bajos precios derivados del comercio internacional. Condiciones no óptimas para el productor.

Los productores ante la incertidumbre y el riesgo optan por entrar a la modalidad de la agricultura por contrato la cual implica costos de transacción, pero ante el riesgo y la incertidumbre deciden afrontarlos para poder de esta manera ser partícipes del mercado en que operan, entonces es aquí cuando adquieren valor los intermediarios financieros, pues sus costos son más bajos que los del mercado y a ante los fallos del mercado, estos pueden ser parte del mercado que les permite ejercer para poder reducir riesgo e incertidumbre antes el medio, para poder realizar de manera eficiente la comercialización (Mahoney, 2012). También existe el aseguramiento del campo en el cual, sin embargo, puede presentarse el riesgo por parte de los productores en el cual el productor puede tener un comportamiento distinto al que venía manejando antes de contar con el seguro agrícola, motivos que pueden hacer que el sector agrícola sufra de fallas del mercado, y que en repetidas ocasiones a través de la historia mexicana se han venido realizando.

Entre las fallas de mercado que se pueden generar por un selección adversa y riesgo moral derivados de la asimetría de la información y por lo tanto falta de las externalidades positivas, que lo que hacen en realidad es elevar los costos de transacción con lo que operan los mercados, entonces en estos casos y como se ha mencionado con anterioridad la intervención del Estado será bien vista, para llevar a cabo las reglamentaciones necesarias que ayuden a las instituciones a operar correctamente, sin dejar de lado aquellos mercados estratégicos, como en esta caso lo es el mercado del maíz, pues es parte de la seguridad nacional del pueblo mexicano.

\section{Referencias}


Akerlof, G. A. (1970). The market for 'lemons': Qualitative uncertainty and the market mechanism. Quarterly Journal of Economics, 84: 488-500.

Arrow, K. J. (1974). The Limits of Organization. New York, NY: W. W. Norton and Company.

Arrow, K. J. (1971). Essays in the Theory of Risk-Bearing. New York, NY: North-Holland Publishing Company.

Camiro Pérez, M. A., Altamirano Cárdenas, R., \& Rojas Herrera, J. J. (2009). Retos del crédito agrícola: estudio de caso de la intermediación financiera en el sur de Sonora, México. Región y sociedad, 21(46), 53-78.

Coase, R. H. (1988). The Firm, the Market and the Law. Chicago: University of Chicago Press.

Coase, R. H. (1960). The problem of social cost. Journal of Law and Economics, 3: 1-44.

Eakin, H., Perales, H., Appendini, K., \& Sweeney, S. (2014). Selling maize in mexico: The persistence of peasant farming in an era of global markets. Development and Change, $45(1), 133-155$.

El Economista. (2014). Importancia y retos actuales del maíz desde Jalisco para México II. Recuperado de http://eleconomista.com.mx/columnas/agronegocios/2014/04/01/importancia-retos-actuales-maiz-jalisco-mexico-ii (MAYO, 2017)

FAO. (1993). El maíz en la nutrición humana. Recuperado de: http://www.fao.org/docrep/T0395S/T0395S02.htm\#Capitulo\%201\%20Introducci\% F3n (Marzo de 2017). Checarlo de las fechas

FIRA. (2016). Quienes somos. Recuperado de: https://www.fira.gob.mx (Mayo, 2017).

FIRA (2015). Panorama Agroalimentario. Recuperado de:

http://www.gob.mx/cms/uploads/attachment/file/200637/Panorama_Agroalimentario_Ma z_2016.pdf

García Salazar, J. A. (2002). Política arancelaria y protección del mercado de maíz en México. Momento Económico, (123), 12-25. doi:http://www.ejournal.unam.mx/cuadros2.php?r=17\&liga=1 
Revista Iberoamericana de Bioeconomia y Cambio Climático (Rev. iberoam. bioecon. cambio clim.) Vol. 3 num 5, 2017, pag 752-768 ISSN eletrónico 2410-7980

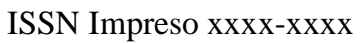

García Salazar, J. A., \& Williams, G. W. (2004). Evaluación de la política comercial de México respecto al mercado de maíz. (with english summary.). El Trimestre Económico, 71(1), 169-213.

Fox, J. and L. Haight (2010). Subsidios para la desigualdad: Las políticas públicas del maíz en México a partir del libre comercio. Washington, DC: Woodrow Wilson International Center for Scholars.

Varian, H. R. (2010). Microeconomía: Un enfoque actual. Barcelona, España: Antoni Bosch, editor.

INEGI (2014). Encuesta Nacional Agropecuaria 2014. Recuperado de: http://www.inegi.org.mx/est/contenidos/proyectos/encuestas/agropecuarias/ena/ena2 014/. (Mayo 2017).

INEGI (2007). Censo Agrícola, Ganadero y Forestal 2007. Recuperado de: http://www.inegi.org.mx/est/contenidos/proyectos/Agro/ca2007/Resultados_Agricol a/ (Mayo,2017).

Mahoney, J. T. (2012). Economic foundations of strategy. Illinois, USA: SAGE

Mahoney, J. T. and Rajendran Pandian, J. (1992). The resource-based view within the conversation of strategic management. Strategic Management Journal, 13: 363-380.

SAGARPA. (2016). Reglas de operación. Recuperado de:

www.sagarpa.gob.mx/agricultura/Programas/proagro/Normatividad/Documents/2017/2016 _12_31_dof_rop_fomento_a_la_agricultura_w.pdf (Mayo 2017).

SAGARPA JALISCO. (2015). Anuarios Estadísticos. Recuperado de http://www.oeidrusjalisco.gob.mx/agricultura/anuarios/index.php

The New York Times ES. (Abril, 2017). México está listo para usar el maíz a su favor en las negociaciones del TLCAN. (2017). Recuperado de: https://www.nytimes.com/es/2017/04/03/mexico-maiz-renegociacion-tlcan/

Vargas Hernández, J. G., Guerra, Bojórquez A., Bojórquez F. (2014). Gestión Estratégica de Organizaciones. Buenos Aires, Argentina: Editorial Elaleph.com 
Revista Iberoamericana de Bioeconomia y Cambio Climático (Rev. iberoam. bioecon. cambio clim.)

Vol. 3 num 5, 2017, pag 752-768

ISSN eletrónico 2410-7980

ISSN Impreso xxxx-xxxx

20 Minutos. (2017). Productores de Tamaulipas aseguran venta de cosechas de maíz y sorgo. Recuperado de http://www.20minutos.com.mx/noticia/203731/0/productores-detamaulipas-aseguran-venta-de-cosechas-de-maiz-y-sorgo/\#xtor=AD-1\&xts=513356. (Marzo, 2017). 